\title{
ADESIVOS TÂNICOS DE Stryphnodendron adstringens (Mart.) Coville NA PRODUÇÃO DE PAINÉIS AGLOMERADOS ${ }^{1}$
}

\author{
Amélia Guimarães Carvalho², Antônio José Vinha Zanuncio², Rafael Farinassi Mendes ${ }^{3}$, Fábio Akira \\ Mori $^{4}$, Marcela Gomes da Silva ${ }^{3}$ e Lourival Marin Mendes ${ }^{4}$
}

\begin{abstract}
RESUMO - O mundo busca fontes alternativas para a produção de adesivos utilizando matéria-prima renovável. Nesse contexto, a utilização de taninos da casca de barbatimão, Stryphnodendron adstringens (Mart.) Coville, tem apresentado potencial. O objetivo deste trabalho foi avaliar a viabilidade técnica de utilização de Adesivos tânicos provenientes das cascas de barbatimão para a produção de painéis aglomerados. Adesivos à base de taninos extraídos das cascas de barbatimão foram usados na proporção de 0, 25, 50, 75 e 100\% com o adesivo comercial ureia-formaldeído. Os adesivos puros e o das misturas tiveram suas propriedades determinadas. Painéis aglomerados com os adesivos ureia-formaldeído, tanino-formaldeído e as misturas destes foram produzidos e suas propriedades físico-mecânicas, testadas. O adesivo tânico de barbatimão mostrou-se viável tecnicamente na proporção de até $50 \%$ para painéis reconstituídos do tipo aglomerado, com a ressalva de recomendação para uso apenas em ambientes internos, sem contato com a umidade.
\end{abstract}

Palavras-chave: Taninos; Barbatimão; Particulados.

\section{TANNIN ADHESIVES FROM Stryphnodendron adstringens (Mart.) Coville IN THE PRODUCTION OF PARTICLEBOARD}

\begin{abstract}
The world seeking alternative sources for adhesives production by using renewable raw material. In this context, the use of Stryphnodendron adstringens (Mart.) Coville tannins bark have been showing potential. The aim of this study was to evaluate the technical feasibility of using tannic adhesive from the barks of barbatimão, for particleboard production. Tannin-based adhesives extracted from the bark of barbatimão were used in ratio of 0, 25, 50, 75 and 100\% with commercial urea formaldehyde adhesive. Pure and mixtures adhesives had their properties determined. Particleboards with urea-formaldehyde, tannin-formaldehyde and its mixtures were produced and had their physic-mechanical properties tested. The adhesive tannic of barbatimão proved be technically feasible in the proportion until 50\% for particleboard panels type, however, it is recommended to indoors use only, without moisture contact.
\end{abstract}

Keywords: Tannins; Barbatimão; Particulate.

\section{INTRODUÇÃO}

A procura por produtos de madeira reconstituída é crescente, tendo em vista que a produção brasileira de painéis aglomerados em 2012 foi de $3.260 .000 \mathrm{~m}^{3}$, aumento de $85 \%$ em relação à produção brasileira em 2000, de 1.762 .000 m³ $^{3}$ FAO - Food and Agriculture
Organization - FAO, 2012). Esse aumento implica maior consumo de adesivos. A ureia-formaldeído (UF) é o principal adesivo utilizado na produção de painéis aglomerados, sendo obtida de fonte não renovável, apresenta intolerância à umidade e possui alta emissão de formaldeído.

\footnotetext{
${ }^{1}$ Recebido em 17.05.2013 aceito para publicação em 03.12.2013

${ }^{2}$ Programa de Pós graduação em Ciência Florestal pela Universidade Federal de Viçosa, UFV, Brasil.E-mail: <ameliagcarvalho@gmail.com> e<ajvzanuncio@yahoo.com.br>.

${ }^{3}$ Programa de Pós Graduação em Ciência e Tecnologia da Madeira pela Universidade Federal de Lavras, UFLA, Brasil. E-mail: <rafael_farinassi@hotmail.com>e <marcela.gsilva@gmail.com>.

${ }^{4}$ Departamento de Ciências Florestais da Universidade Federal de Lavras, UFLA, Brasil. E-mail: <morif@dcf.ufla.br>e $<$ lourival@dcf.ufla.br>.
} 
Por não ser resistente à umidade, o adesivo UF é utilizado em produtos que se destinam a ambientes internos, entretanto o formaldeído emitido por esse adesivo, de acordo com Iark (2004), é um composto cancerígeno, razão por que seu uso nesses locais deve ser evitado.

A busca e desenvolvimento de adesivos alternativos, oriundos de fontes renováveis, com baixa ou nenhuma emissão de formaldeído, são uma realidade. Com destaque para a utilização de taninos condensados, que podem ser utilizados como substitutos de resinas sintéticas, pois precipitam com o formaldeído, formando, assim, um polímero de estrutura rígida. Esses adesivos são denominados taninos-formaldeídos (TF) (PIZZI, 1994).

Os taninos condensados possuem unidades flavonoides com diferentes graus de condensação e alta reatividade com o formaldeído. A alta reatividade decorre do anel A da unidade flavonoide, que pode ser resorcinólico ou floroglucinólico, podendo atingir, em condições similares, velocidade de reação de 10 a 50 vezes superior à da reação do fenol com o formaldeído (PIZZI, 2003).

Os taninos condensados comerciais mais comuns são aqueles oriundos da casca da acácia (Acacia mearnsii ou mollissima), da madeira do quebracho (Schinopsis balansae e Schinopsi lorentzii), da casca de pinus (Pinus radiata) e das folhas de gambir (Uncaria gambir) (TONDI; PIZZI, 2009).

Stryphnodendron adstringens (Mart.) Coville, espécie do Cerrado e conhecida popularmente como barbatimão, apresenta valor econômico para extração de taninos de suas cascas e folhas. O rendimento médio é entre $27 \%$ e $32 \%$ nas folhas, nos meses de maio e setembro (ALMEIDA et al., 2008); e de $40 \%$ na casca (MORI et al., 2006).

Não existem estudos a respeito do adesivo tânico de barbatimão em painéis particulados. Assim, o objetivo desta pesquisa foi avaliar a utilização de adesivo à base de taninos provenientes da casca de Stryphnodendron adstringens (Mart.) Coville, na produção de painéis aglomerados.

\section{MATERIAL E MÉTODOS}

\subsection{Obtenção do adesivo tânico de barbatimão}

As cascas de barbatimão foram coletadas em área de Cerrado, localizada no Município de Lavras, MG.
Após a coleta, as cascas foram misturadas, fragmentadas, secas ao ar livre e moídas em moinho tipo martelo, sendo separadas com uma peneira de $2 \mathrm{~mm}^{2}$ de abertura.

A extração dos polifenóis do Stryphnodendron adstringens (Mart.) Coville foi realizada com a utilização de $3 \%$ de sulfito de sódio em relação à massa seca, a $70{ }^{\circ} \mathrm{C}$, durante $3 \mathrm{~h}$, empregando-se uma relação licor/ casca de 15:1 v:m. Após a extração, o material foi filtrado em peneira de malha de $1 \mathrm{~mm}^{2} \mathrm{e}$, em seguida, em cadinhos de vidro sinterizados de porosidade 2 , acoplados a uma bomba de vácuo. Os extratos foram distribuídos em bandejas de vidro e submetidos a $40^{\circ} \mathrm{C}$ até a secagem total. Em seguida, foram moídos manualmente com a utilização de um graal de porcelana com pistilo.

Os adesivos termofixos à base de taninos foram sintetizados com 50\% de sólidos totais em um agitador, com rotação de 3.000 RPM. Após 24 h de hidratação, foram adicionados $8 \%$ do paraformaldeído (agente ligante) em relação à massa seca de taninos em pó.

\subsection{Propriedades dos adesivos}

Foram determinadas as propriedades de viscosidade, gel time, teor de solidos e pH dos adesivos taninoformaldeído e ureia-formaldeído, assim como das misturas desses dois adesivos.

A viscosidade foi determinada utilizando um viscosímetro do tipo Copo Ford (Universal), seguindo-se os parâmetros estipulados pela norma ASTM D-1200 (ASTM, 1994).

O tempo de gel foi obtido utilizando-se amostras de 5 g de adesivo de cada tratamento, colocadas em tubos de ensaio de $15 \mathrm{~cm}$ de altura e $2 \mathrm{~cm}$ de diâmetro. O conjunto tubo-bastão foi mergulhado em um banho de glicerina na temperatura de $130{ }^{\circ} \mathrm{C}$. Com o auxílio do bastão, o líquido foi constantemente agitado com movimento vertical, em razão do aumento na resistência do adesivo ao atingir a "fase de gel”. O tempo de gelatinização de cada tratamento foi aquele entre imersão do tubo na glicerina e o momento em que a amostra endureceu, sendo correspondente à polimerização ou gel time do adesivo.

A determinação do teor de sólidos foi realizada pesando-se $1 \mathrm{~g}$ do adesivo, secando-o na estufa à temperatura de $103 \pm 3{ }^{\circ} \mathrm{C}$, por $3 \mathrm{~h}$. O percentual do teor de sólidos foi calculado pela divisão da massa final pela massa inicial vezes 100 . 
$\mathrm{O}$ pH dos extratos aquosos foram determinados pelo pH-metro, após 4 min de contato com o adesivo.

\subsection{Produção dos painéis aglomerados}

A determinação da densidade básica da madeira de Pinus oocarpa foi realizada de acordo com a norma NBR 11941 (NBR 11941 - ABNT, 2003).

Para a produção dos painéis foram considerados cinco tratamentos, sendo para cada tratamento produzidos três painéis. Os painéis foram produzidos com o adesivo comercial ureia-formaldeído (T1) e nas proporções de 25 (T2), 50 (T3), 75 (T4) e 100\% (T5) de adesivo tânico de barbatimão em relação ao adesivo UF.

As toras de Pinus oocarpa foram aquecidas em um tanque com água a $66^{\circ} \mathrm{C}$ por $24 \mathrm{~h}$, processadas em torno laminador com espessura nominal de $2 \mathrm{~mm}$ e submetidas ao moinho de martelo para obtenção das partículas. Estas foram processadas em peneira vibratória, secas ao ar livre até atingirem umidade entre 12 e 15\% e secas em uma estufa de ventilação forçada a uma temperatura de $103 \pm 2{ }^{\circ} \mathrm{C}$ até atingir a umidade em torno de $3 \%$.

Para cada tratamento foram produzidos painéis com densidade nominal de 0,65 g. $\mathrm{cm}^{-3}$. Foi aplicado às partículas o adesivo no teor de $8 \%$ (base massa seca das partículas), por meio de aspersão, em uma encoladeira do tipo tambor giratório.

Uma vez impregnadas, as partículas foram levadas para uma caixa formadora de colchão, com dimensões de $48 \mathrm{~cm}$ x $48 \mathrm{~cm}$, e submetidas a uma pré-prensagem, a $4 \mathrm{kgf} / \mathrm{cm}^{2}$, sendo, então, levado à prensa quente, com separadores de $15 \mathrm{~mm}$ de espessura, onde passaram por um ciclo de prensagem de $8 \mathrm{~min}$, a $160^{\circ} \mathrm{C}$ e $40 \mathrm{kgf} . \mathrm{cm}^{-2}$.

\subsection{Avaliação das propriedades físico-mecânicas}

A absorção de água (AA) após 2 e 24 h de imersão, inchamento em espessura (IE) após 2 e 24 h de imersão e taxa de não retorno em espessura (TNRE) foram realizados conforme ASTM D 1037 (2006). Para avaliação das propriedades MOR e MOE à flexão estática, foi empregada a Norma DIN 52362 (1982), enquanto para a densidade e ligação interna, a Norma ASTM D 1037/2006.

\subsection{Análise estatística}

Foi considerado o delineamento inteiramente casualizado (DIC). Para diferenciação entre as médias dos tratamentos, foi realizada a análise de variância a 5\% de significância, seguida de análise de regressão em nível de significância de 5\%. Somente para a diferenciação entre as densidades médias de cada tratamento foi utilizado o teste de média de Tukey a 5\% de significância.

O teste de homogeneidade de variâncias (teste de Bartlett a 5\% de significância) e o de normalidade (teste de Shapiro-Wilk a 5\% de significância) foram utilizados para analisar a dispersão dos dados.

\section{RESULTADOS}

\subsection{Propriedades dos adesivos}

A viscosidade do adesivo foi de 494 cP e 218 cP para adesivo tânico e da ureia-formaldeído, enquanto as proporções 25, 50 e $75 \%$ de adesivos tânicos apresentaram 270, 319 e 380 cP, respectivamente.

O tempo de gel apresentou tendência inversa, com 90s para adesivo tânico e 120s para UF. A proporção de 25, 50 e 75\% de adesivo tânico apresentou valores de 53,55 e 61 s, respectivamente.

O teor de sólidos dos adesivos foram próximos de $50 \%$. Os valores de 0 , 25, 50, 75 e $100 \%$ pelo adesivo tânico de barbatimão foram de 53,8; 52,2; 50,6; 52,7; e $50,7 \%$, respectivamente.

$\mathrm{O}$ pH do adesivo UF foi 8,9, enquanto o do adesivo tânico, 4,9; o aumento da concentração de adesivos tânicos diminuiu o pH, e as proporções de 25, 50 e $75 \%$ apresentaram valores de 6,0; 5,2; e 5,1, respectivamente.

\subsection{Densidade aparente e razão de compactação dos painéis}

A densidade básica da madeira do Pinus oocarpa foi de $0,42 \mathrm{~g} \cdot \mathrm{cm}^{-3}$. Não houve diferença na densidade dos painéis pelo teste de Tukey a 5\% de significância, nos diferentes tratamentos. A densidade aparente foi de 0,$597 ; 0,598 ; 0,583 ; 0,593 ; 0,588$ g. $\mathrm{cm}^{-3}$ nos painéis produzidos com o adesivo UF e a substituição deste pelo adesivo tânico de barbatimão a 25, 50, 75 e 100\%, respectivamente. A razão de compactação de todos os tratamentos foi da ordem de 1,4.

\subsection{Absorção de água}

A absorção de água média após 2 e 24 h nos tratamentos com a UF e a substituição desse adesivo pelo adesivo tânico nas proporções de 25, 50 e 75\%



Revista Árvore, Viçosa-MG, v.38, n.1, p.195-202, 2014 


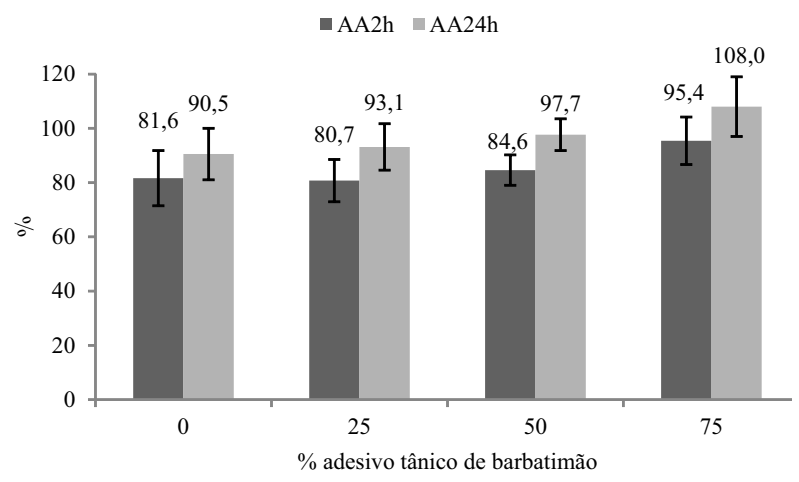

Figura 1 - Absorção de água após 2 e 24 h de imersão em água dos painéis aglomerados.

Figure 1 - Water absorption of the particleboards after 2 and 24 hours of immersion in water.

estão representadas na Figura 1. Não houve relação significativa com o aumento da substituição do adesivo ureia-formaldeído pelo adesivo tânico de barbatimão.

Apesar de não haver tendência significativa, os valores aumentam com a elevação da proporção de adesivo tânico. Entretanto, o tratamento com 100\% de adesivo tânico de barbatimão não foi resistente à água, impossibilitando a medição das propriedades físicas desse tratamento.

\subsection{Inchamento em espessura}

Houve relação significativa na regressão linear para o inchamento em espessura após 2 e 24 h (Figura 2), com valores crescentes com o aumento da proporção de adesivo tânico.

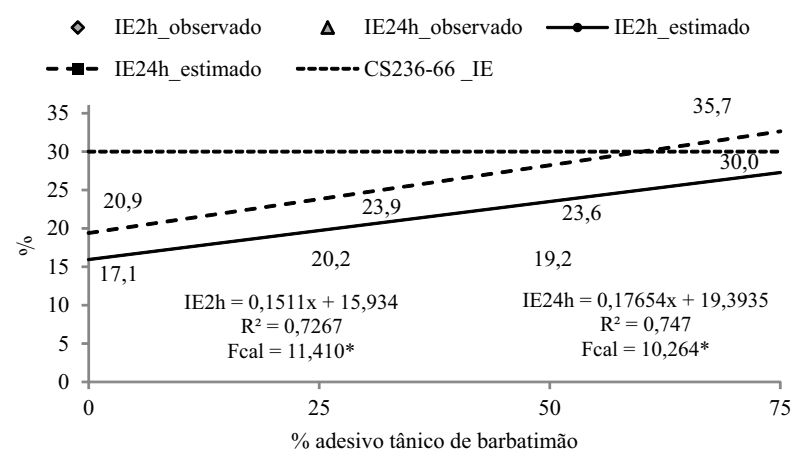

Figura 2 - Inchamento em espessura após 2 e 24 h de imersão em água dos painéis aglomerados. * Significativo a $5 \%$.

Figure 2 - Swelling in thickness of the particleboard after 2 and 24 hours of immersion in water.*Significant at the level of $5 \%$.

\subsection{Ligação interna}

A regressão quadrática foi a que melhor representou a relação entre porcentagem de adesivo tânico adicionado ao adesivo UF e a propriedade ligação interna (LI) dos painéis aglomerados, sendo esta estatisticamente significativa (Figura 3). Houve redução dos valores médios de ligação interna à medida que se elevou a proporção de adesivo tânico.

\subsection{Módulo de ruptura e de elasticidade à flexão estática}

A relação linear entre o MOR e a substituição do adesivo UF pelo adesivo tânico de barbatimão se mostrou significativa (Figura 4), assim como também foi significativa a relação linear entre o MOE e a porcentagem de adesivo tânico utilizado (Figura 5). O aumento da substituição do adesivo UF pelo tânico reduziu os valores de MOE e MOR dos painéis aglomerados.

\section{DISCUSSÃO}

\subsection{Propriedades dos adesivos}

Devido à maior viscosidade do adesivo à base de taninos de barbatimão em relação à ureia-formaldeído (UF), a adição deste aumentou a viscosidade, contudo em padrões aceitáveis para aplicação por aspersão na produção de painéis particulados.

O menor tempo de gel do adesivo tanino mostra a elevada reatividade dos taninos em relação ao paraformaldeído, o que promove a cura mais rápida do adesivo e reduz a sua vida útil. A redução dos valores de gel time das misturas dos adesivos, pode ser devido ao elevado $\mathrm{pH}$ da cola UF, uma vez que o adesivo de

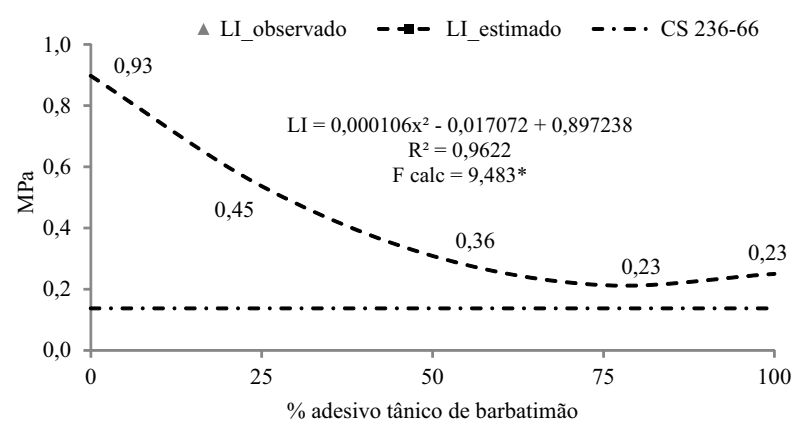

Figura 3 -Ligação interna dos painéis aglomerados. * Significativo a $5 \%$.

Figure 3 - Internal bond of the particleboard *significant at the level of 5\% significance. 


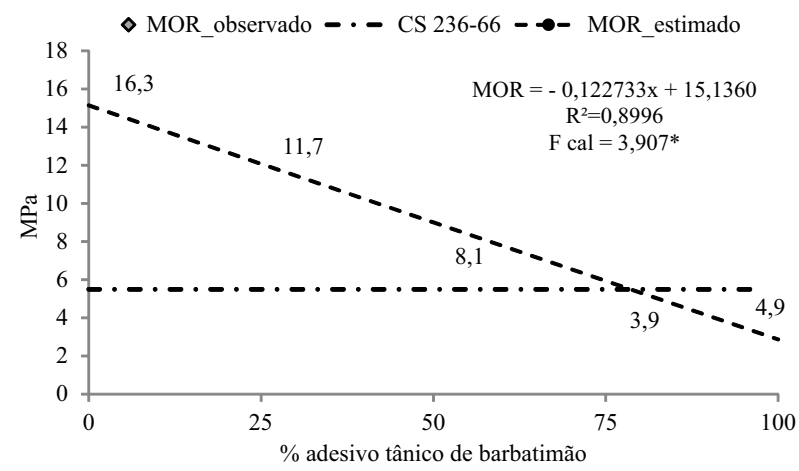

Figura 4 - Módulo de ruptura dos painéis aglomerados. *Significativo a $5 \%$.

Figure 4-Mean values of MOR of the particleboard *significant at the level of $5 \%$ significance.

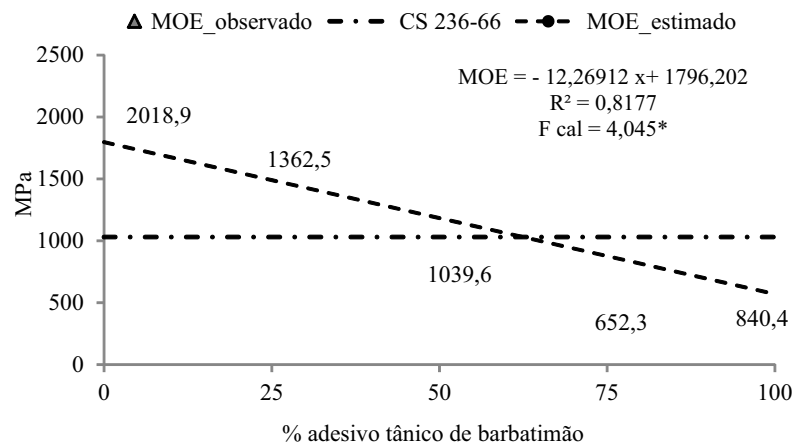

Figura 5 - Módulo de elasticidade dos painéis aglomerados. * Significativo a $5 \%$.

Figure 5-Mean values of MOE of the particleboard. *Significant at the level of $5 \%$ significance.

tanino em meio com $\mathrm{pH}$ elevado, é polimerizado mais rápido.

O teor de sólidos indica a porcentagem de sítios reativos com o agente ligante, e maior teor de sólidos do adesivo torna mais resistente a sua linha de cola. No entanto, altos teores de sólido prejudicam a aplicação do adesivo via aspersão, devido ao aumento da sua viscosidade, assim como também dificulta o espalhamento e penetração dos adesivos nas lâminas de madeira para produção de compensados.

Os taninos foram hidratados para obtenção de adesivo com teor de sólidos de 50\%, faixa adequada para produção dos painéis particulados. Todos os adesivos produzidos apresentaram valores próximos a esse.
O adesivo tânico é ácido, enquanto a ureiaformaldeído é básica. Conforme houve aumento da substituição do adesivo UF pelo adesivo tânico de barbatimão, o pH das misturas apresentaram redução de seus valores.

\subsection{Densidade aparente e razão de compactação}

De acordo com a norma comercial americana CS 236-66 (COMMERCIAL STANDARD, 1968), todos os painéis aglomerados deste trabalho se enquadram no tipo 1 (uso interior) e densidade C (abaixo de 0,600 g.cm³). Já em relação à razão de compactação, todos os tratamentos estão dentro da faixa estipulada como adequada por Maloney (1993), que é de 1,3 a 1,6.

\subsection{Absorção de água}

Os valores de absorção de água, após 2 e 24 h de imersão, obtidos neste estudo, apresentaram-se acima dos encontrados na literatura para adesivos tânicos de outras espécies.

Carneiro et al. (2009) produziram painéis aglomerados com densidade de 0,67 g. $\mathrm{cm}^{-2}$, utilizando o adesivo UF e a substituição deste em $25 \%$ pelo adesivo tânico de angico-vermelho, com o teor de $8 \%$ de adesivo em relação à massa seca de partículas. Esses autores encontraram valores de absorção de água de 72 e 57\% para AA2h e 89 e 77\% para AA24h, para o adesivo UF e a substituição em 25\%, respectivamente.

Gonçalves et al. (2003) confeccionaram painéis aglomerados com partículas de Eucalyptus pellita utilizando o adesivo UF e a substituição deste em 10 e 20\% pelo adesivo tânico de Mimosa caesalpiniaefolia, sendo os taninos extraídos utilizando $0,5 \%$ de sulfito de sódio (relação à massa seca das partículas) como extrator. Aqueles autores aplicaram 10\% de adesivo em relação à massa seca das partículas, e a densidade encontrada foi de 0,59 g. $\mathrm{cm}^{-3}$ para o adesivo UF e 0,61 e 0,60 g. $\mathrm{cm}^{-3}$ para a substituição em 10 e 20\%, respectivamente. Para AA2h, os mesmos autores encontraram valores de 69,0; 73,3; e 74,4\% e para AA24h, valores de 81,$4 ; 85,5$; e $85,1 \%$, nos tratamentos com UF e na substituição de 10 e $20 \%$, respectivamente.

\subsection{Inchamento em espessura}

Houve aumento do inchamento em espessura dos painéis aglomerados com o incremento da proporção de adesivos tânicos de barbatimão. 
Carneiro et al. (2009), produzindo painéis aglomerados com partículas de Pinus elliotti, densidade de 0,67 g. $\mathrm{cm}^{-3}$, encontraram valores de IE2h de 19,6; 15,2; e 29,7\% e de IE24h de 25,1; 20,5; e 56,4\% para os painéis com adesivo UF, com 25\% de substituição e painéis produzidos apenas com o adesivo tânico, respectivamente.

Gonçalves et al. (2008) produziram painéis aglomerados com a madeira de Eucalyptus urophylla, densidade aparente média de 0,60 g. $\mathrm{cm}^{-3}$ e $10 \%$ de adesivo UF (base massa seca das partículas) sem e com a substituição deste em $10 \%$ pelo adesivo tânico comercial de Acacia mearnsii. Esses autores obtiveram valores médios de IE 2 h de 20,1 e 20,8\% e de IE24 h de 27,8 e $26,5 \%$ para os painéis apenas com UF e com UF mais $10 \%$ de tanino, respectivamente.

O inchamento em espessura máximo permitido após 24 h de imersão é de 30\% (CS 236-66 COMMERCIAL STANDARD, 1968). Os painéis produzidos com $75 \%$ e $100 \%$ de tanino ultrapassaram esse valor. Desse modo, apenas os teores de 25 e 50\% de adesivo de tânico de barbatimão podem ser utilizados para produção de painéis aglomerados sem que ocorra prejuízo nessa propriedade.

\subsection{Ligação interna}

A ligação interna deve ultrapassar 0,14 MPa em painéis com adesivo ureia-formaldeído e de baixa densidade (CS 236-66 - COMMERCIAL STANDARD, 1968). Ping et al. (2011) testaram a ligação interna de painéis aglomerados confeccionados com adesivo tânico extraídos de bagaço de uva. Os painéis foram produzidos com adesivo de $10 \%$ de sólidos (baseado no peso das partículas secas) e com a densidade aparente de 0,69 g.cm-3. Esses autores encontraram valor de resistência à ligação interna de 0,26 MPa nos painéis com 100\% de adesivo tânico de bagaço de uva.

Carneiro et al. (2009) produziram painéis aglomerados com partículas de Pinus elliotti, densidade de $0,67 \mathrm{~g} . \mathrm{cm}^{-3}$ e com adesivo UF e o adesivo tânico de angico-vermelho. Esses autores encontraram valores médios de 0,54 MPa para o tratamento com adesivo tânico de angico-vermelho a 0,78 MPa nos painéis produzidos com o adesivo UF.

Assim, os valores obtidos para ligação interna neste trabalho foram inferiores aos encontrados na literatura, porém, em relação à norma supracitada, todos os tratamentos atenderam ao mínimo estipulado.

\subsection{Módulo de ruptura e de elasticidade à flexão estática}

O módulo de ruptura (MOR) e o módulo de elasticidade (MOE) tiveram seus valores reduzidos com o aumento da proporção de adesivo tânico.

Carneiro et al. (2009) produziram painéis aglomerados com partículas de Pinus elliotti, densidade aparente média de 0,67 g.cm ${ }^{-3}$ e com adesivo UF na substituição de 25 e 100\% pelo adesivo tânico de angico-vermelho. Esses autores encontraram valores médios variando para o MOR de 19,96; 18,25; e 17,11 MPa e para o MOE de 2329; 1659 e $1924 \mathrm{MPa}$, nos adesivos UF e 25 e $100 \%$ de substituição pelo adesivo de angico, respectivamente.

Guimarães Júnior et al. (2011) produziram painéis aglomerados utilizando três clones de E. saligna, com 8\% de adesivo UF a 8\% (base massa seca das partículas). Os painéis obtiveram densidade média de 0,62 g.cm-3 e valores entre 654,1 e 762,4 MPa para MOE e entre 12,5 e 16,1 MPa para o MOR.

Gonçalves et al. (2008) produziram painéis aglomerados com a madeira de Eucalyptus urophylla, densidade aparente média de 0,60 g. $\mathrm{cm}^{-3}$ e $10 \%$ de adesivo UF (base massa seca das partículas), assim como a substituição deste em $10 \%$ pelo adesivo tânico comercial de Acacia mearnsii. Esses autores obtiveram valores médios de MOR de 13,5 e 12,9 e de MOE, valores médios de 1.887 e $1.815 \mathrm{MPa}$, para os painéis com o adesivo UF e o adesivo UF mais 10\% de adesivo tânico de acácia, respectivamente.

A Norma de Comercialização CS 236-66 (COMMERCIAL STANDARD, 1968) estipula como valor mínimo, para painéis de baixa densidade e adesivo ureiaformaldeído, os valores de 5,49 MPa para o MOR e de 1029,7 MPa para o MOE.

Nesse sentido, em relação aos dados obtidos na literatura, apenas os resultados observados para os painéis produzidos com 100\% de ureia-formaldeído apresentaram valores médios próximos. No entanto, em comparação com a norma de comercialização, apenas os painéis produzidos com 100 e $75 \%$ de tanino não a atenderam. Podem, assim, ser utilizados os teores de 25 e 50\% de substituição de UF pelo adesivo de 
tanino à base de barbatimão para produção de painéis aglomerados, sem que ocorra prejuízo das propriedades MOR e MOE à flexão estática.

\section{CONCLUSÕES}

A substituição de adesivo ureia-formaldeído por adesivo de tanino de barbatimão na produção de painéis aglomerados não apresentou efeito significativo apenas nas propriedades de absorção de água após 2 e 24 h de imersão. Não se levaram em consideração, nesses dois casos, os painéis com $100 \%$ de adesivo à base de tanino, visto que eles não resistiram ao contato com a água.

Nas demais propriedades, o incremento na porcentagem de tanino promoveu o aumento dos valores do inchamento em espessura após 2 e 24 h de imersão e a diminuição dos valores de ligação interna, módulo de ruptura e módulo de elasticidade à flexão estática.

Os painéis aglomerados com até 50\% de adesivo tânico de barbatimão se mostraram, no entanto, aptos para utilização, atendendo a todos os prerrequisitos estipulados pela norma de comercialização.

O adesivo tânico de barbatimão mostrou-se viável tecnicamente para aplicação parcial em painéis reconstituídos do tipo aglomerado. Recomenda-se seu uso apenas em ambientes internos, sem contato com a umidade, mas não o uso do adesivo puro em até 50\% de substituição do adesivo UF.

\section{AGRADECIMENTOS}

À Fundação de Amparo à Pesquisa do Estado de Minas Gerais (FAPEMIG), à Coordenação de Aperfeiçoamento de Pessoal de Nível Superior (CAPES) e ao Conselho Nacional de Desenvolvimento Científico e Tecnológico (CNPq), pelo apoio financeiro.

\section{REFERÊNCIAS}

ALMEIDA, N. F. et al. Rendimentos em taninos das folhas de barbatimão [Stryphnodendron adstringens] em diferentes períodos de coleta visando a produção de adesivo para a madeira. In: ENCONTRO BRASILEIRO EM MADEIRA E ESTRUTURAS DE MADEIRA, 11., 2008, Londrina. Anais... Londrina: UEL, 2008. p.5-8.

\author{
AMERICAN SOCIETY FOR TESTING AND \\ MATERIALS - ASTM D-1037: standard \\ methods of evaluating properties of wood- \\ base fiber and particles materials. \\ Philadelphia: 2006.
}

\section{AMERICAN SOCIETY FOR TESTING AND \\ MATERIALS - ASTM D 1200: Test method for} Viscosity by Ford Viscosity. Philadelphia: 1994.

\section{ASSOCIAÇÃO BRASILEIRA DE NORMAS TÉCNICAS - ABNT. NBR 11941. Brasília: 2003. 6 p.}

CARNEIRO, A. C. O. et al. Properties of particleboards glued with adhesives of "angicovermelho" tannin (Anadenanthera peregrina) and urea-formaldehyde. Revista Árvore, v.33, n.3, p.521-531, 2009.

\section{COMMERCIAL STANDARD. Mat formed wood particleboard: CS 236-66. [S. 1.: s. n.], 1968.}

\section{DIN - NORMEN FÜR HOLZFASERPLATEN SPANPLATTEN SPERRHOLZ. DIN 52362 - Testing of wood chipboards, bending test, determination of bending strength. Germany: 1982. p.39-40.}

FOOD AND AGRICULTURE ORGANIZATION FAO. Disponível em: <http://faostat.fao.org/site/ 630/default.aspx>. Acesso em: 24 de out. de 2013.

GONÇALVES, C. A. et al. Produção de chapas de madeira aglomerada com adesivo Uréiaformaldeído modificado com tanino de mimosa Caesalpiniaefolia Bentham (Sabiá). Floresta e Ambiente, v.10, n.1, p.18-26, 2003.

GONÇALVES, C. A.; LELIS, R. C. C.; OLIVEIRA, J. T. S. Influência da composição da resina taninouréia-formaldeído Nas propriedades físicas e mecânicas de chapas aglomeradas. Revista Árvore, v.32, n.4, p.715-722, 2008.

GUIMARÃES JÚNIOR, J. B. et al. Painéis de madeira aglomerada de resíduos da laminação de diferentes procedências de Eucalyptus grandis, Eucalyptus saligna e Eucalyptus cloeziana. Cerne, v.17, n.4, p.443-452, 2011.



Revista Árvore, Viçosa-MG, v.38, n.1, p.195-202, 2014 
INTERNATIONAL AGENCY FOR RESEARCH ON CANCER - IARC. Iarc classifies

formaldehyde as carcinogenic to humans. 2004. (Press Release, n. 153).

Disponível em: <http://www.iarc.fr/en/mediacentre/pr/2004/pr153. html>. Acesso em: 22 ago. 2012.

\section{MALONEY, T. M. Modern particleboard} \& dry process fiberboard

manufacturing. San Francisco: M. Freeman, 1993.681p.

MORI, F. A. et al. Quantificação de taninos das cascas de barbatimão (Stryphnodendron adstringens). In: ENCONTRO BRASILEIRO EM MADEIRA E ESTRUTURAS DE MADEIRA, 10., 2006, São Pedro. Anais... São Pedro: [s. n.], 2006.
PING, L. et al. Condensed tannins extraction from grape pomace: characterization and utilization as wood adhesives for wood particleboard.

Industrial Crops and Products, v.34, p.907-914, 2011.

PIZZI, A. Tannin-based wood adhesives. In: PIZZI, A. Advanced wood adhesives technology. New York: M. Dekker, 1994. p.149-217.

PIZZI, A. Natural phenolic adhesive I: tannin. In: PIZZI, A.; MITTAL, K. L. (Org.). Handbook of adhesive technology. 2.ed. New York: $M$. Dekker, 2003. p.573-587.

TONDI, G.; PIZZI, A. Tannin-based rigid foams: Characterization and modification. Industrial Crops and Products, v.29, p.356-363, 2009. 